\title{
BITCOIN AND THE CROSS-MARKET EFFECTS OF THE MT. GOX MELTDOWN
}

\author{
Michael Williams, Governors State University, mwilliams15@govst.edu \\ Semih Emre Çekin, Turkish-German University, scekin@tau.edu.tr \\ David Green, Governors State University, dgreen@govst.edu
}

\begin{abstract}
Bitcoin is a well known cryptocurrency that has existed for over a decade. We examine the historical cross-market dynamic relationships among four important Bitcoin cryptocurrency markets at a pivotal point in cryptocurrency acceptance among markets and the public. We pay particular attention to cross-market relations during the introduction of a new, competing Bitcoin exchange, Bitfinex, and the subsequent demise of the once-prominent Bitcoin exchange, Mt. Gox. Our findings show that Bitfinex's introduction led to a shifting of trading activity from the once popular Mt. Gox exchange to other exchanges. Mt. Gox's loss of trading activity caused price distortions in all Bitcoin markets under study. In addition, cross-market relationships became less efficient while Mt. Gox experienced its death throes. Our study provides evidence that (1) Bitcoin markets are susceptible to volume losses to rival exchanges, (2) shifting trading activity is also associated with price distortions mainly originating from the suffering exchange, and (3) Bitcoin cross-market dynamics are resilient and, ultimately, self-healing from shocks, strengthening Bitcoin's long-term viability.
\end{abstract}

Keywords: Bitcoin; cryptocurrency; virtual currency

\section{INTRODUCTION}

Since its inception in 2009, Bitcoin has become a widely discussed electronic payment system around the globe. Bitcoin exchanges have played an important role advancing the viability of Bitcoin but have also hurt Bitcoin's image due to a series of problems that occurred among exchanges. Of the exchanges on which the virtual currency or cryptocurrency has traded, Mt. Gox, a Japanese based exchange for cryptocurrency, played an especially important role. Until the first half of 2013, the vast majority of trading volume for Bitcoin occurred on the Mt. Gox exchange whereas other exchanges played a negligible role. The decline occurred in 2013, and by December 2013, Mt. Gox accounted only for one third of all trading. By March 2014, Mt. Gox was shut down and all operations and trades ceased. The substantial trade vacuum that came as a result of Mt. Gox's termination was quickly absorbed by three exchanges, Bitfinex, Bitstamp and BTC-e.

Our paper adds to the cryptocurrency discussion by examining exchanges that trade Bitcoin and the effects of the Mt. Gox Bitcoin exchange meltdown, including its causes and the response of the Bitcoin market once Mt. Gox was no more.

\section{LITERATURE REVIEW}

Bitcoin is a bidirectional virtual currency, which is a subcategory of the broader digital currency category. Digital currencies include both electronic money and virtual currencies. Electronic money is one type of digital money using traditional currency with legal tender status (i.e., USD, Euro, etc.) as the unit of account. Bitcoin exchanges typically trade in one or more currencies. This paper focuses on exchanges trading in the US Dollar (USD) to Bitcoin (BTC).

Prior to late 2013, Mt. Gox was the leader in USD/BTC trading. Founded in 2010, by 2013, Tokyo-based Mt. Gox exchange handled up to $80 \%$ of all Bitcoin trading volume (Abrams \& Popper, February 25, 2014). However, this dominant position of Mt. Gox was short-lived as frequent distributed denial-of-service (DDoS) attacks, price manipulation, stolen Bitcoin and other problems resulted in Mt. Gox's move toward insolvency from the middle of 2013 until trades halted for good on February 25, 2014 (see e.g. Feder et al., 2017 or Gandal et al., for problems in the Bitcoin ecosystem). 


\section{Issues in Information Systems}

Volume 21, Issue 3, pp. 245-252, 2020

In the period after Mt. Gox trading ceased in 2014, USD to BTC trading was largely concentrated among three exchanges, Bitstamp, BTC-e, and Bitfinex, with the three exchanges holding $71 \%$ of USD/BTC trading volume from June to December 2014. Bitstamp is a cryptocurrency exchange founded in Slovenia in 2011, operating in the United Kingdom since 2013 (Hill, 2014). Over a six-month period, Bitstamp had 24.48\% of USD/BTC trading volume. Bitfinex is one of the largest cryptocurrency trading platforms accepting USD deposits and trades. Over a six-month period, Bitfinex had 34.7\% of USD/BTC trading volume. The exchange's servers are located in the United Kingdom. BTC-e is believed to be based in Bulgaria, and according to the exchange's website it operates under the jurisdiction of Cyprus (BTC-e). Over a six-month period, BTC-e had 12.3\% of USD/BTC trading volume.

While characteristics of Bitcoin as a virtual currency and the reasons for the demise of the Mt. Gox exchange were previously analyzed by various authors (see e.g. Böhme et al., 2015 and Feder et al., 2017), the literature on crossmarket dynamics remains inconclusive or scant.

To fill this gap, this paper explores the impact of market conditions and external events on Bitcoin and the crossmarket relationships of Bitcoin exchanges, specifically focusing on events including the collapse of the Mt. Gox exchange and introduction of new exchanges. Understanding the cross-market effects of specific conditions and market events provides insight into the resiliency of Bitcoin and Bitcoin markets along with shedding light on the long-term viability of the cryptocurrency.

\section{RESEARCH METHODOLOGY}

In order to understand the evolution of Bitcoin market dynamics across various "market regimes", we use a split sample approach. Specifically, for each distinct set of market conditions, we calculate our contemporaneous and causality metrics and then compare said metrics among the various, distinctly-estimated regimes. In doing so, we gain insights regarding how certain market conditions or external events impacted the various cross-Bitcoin relationships.

Our sample includes price data from Bitcoincharts.com and volume data from Bitcoinity.org and covers all days between July 17th, 2010 to September 30th, 2014 where each relevant Bitcoin market reported a closing price estimate. We select four of the largest Bitcoin markets during this overall period for our study: Bitstamp, BTC-e, Bitfinex, and Mt. Gox. Our initial date was selected based on when data was first available for Mt. Gox.

Our end date was selected based on a cost-benefit analysis of choosing the longest dataset possible without incorporating spurious events into the analysis. The Bitcoin market ecosystem has been in a state of constant flux over the last few years with multiple market entrances and disturbances since the fall of Mt. Gox. While we would like to incorporate as much data as possible and, in the process, lower estimation uncertainty, we opted for the September 30th 2014 end data to prevent our analysis from being biased by non-Mt. Gox-related market events.

Based on the full sample, we created three sub-samples that correspond to various major Bitcoin market regimes. The first sub-sample ("Pre-Bitfinex") corresponds to a time when Mt. Gox, BTC-e, and Bitstamp were in operation but where trading on Bitfinex had not yet commenced. The Pre-Bitfinex sample spans September 13th, 2011 to March 30th, 2013, comprises 565 mutually-available observations, and ends just before the introduction of the Bitfinex exchange. Thus, this first sub-sample represents a fairly tranquil period when three major Bitcoin exchanges were in operation and before major market events had occurred.

The second sub-sample ("All Markets") corresponds to the time when all four of the study's major exchanges were in operation: Mt. Gox, BTC-e, Bitstamp, and Bitfinex. Specifically, the All Markets sub-sample spans March 31st, 2013 to February 25th, 2014, begins the first day that Bitfinex data was available, ends on the last day Mt. Gox data is available (i.e. the day of its Mt. Gox's demise), and comprises a total of 332 mutually-available observations. The All Markets sub-sample thus captures two major events within the Bitcoin market ecosystem: the introduction of Bitfinex and the demise of Mt. Gox.

We also constructed a third sub-sample ("Mt. Gox Gone") spanning February 26th, 2014 to September 30th, 2014 and comprising 215 mutually-available observations to shed light on the motivating factors of dysfunction in Bitcoin markets. During the Mt. Gox Gone sub-sample, only three Bitcoin markets remain: BTC-e, Bitstamp, and Bitfinex. 


\section{Issues in Information Systems \\ Volume 21, Issue 3, pp. 245-252, 2020}

Throughout our study, we define the Weighted Bitcoin Price as the daily transaction-volume-weighted average price across all available exchanges. We define individual Price Divergence as the absolute percentage difference in an individual-market's closing Bitcoin price relative to the closing Weighted Price. To analyze an aggregate measure of Bitcoin market dysfunction we define Total Bitcoin Pricing Error as the sum of each market's individual Price Divergence value (where data available). In order to analyze each market's relative market power, we define a market's Trade Share as the total daily trading in Bitcoins on that market divided by the total amount of Bitcoins trading on all (open) markets. Finally, we define a Bitcoin market's return as the percentage difference in close-toclose $\log$ price. Tables 1 and 2 report summary statistics for the price and volume data that we utilize.

In addition to our use of descriptive analyses for Bitcoin pricing error (or distortions), trading activity, and trade shares, we also employ two other methodologies to study the cross-market dynamics among each sub-sample's Bitcoin market conditions. The first is an unconditional contemporaneous correlation analysis using 30-day rollingwindow samples. During times where Bitcoin markets function well, we should expect to see a fairly high degree of cross-market contemporaneous correlation between each market pair given that each market provides a (mostly) fungible cryptocurrency product and that any major cross-market distortions should be arbitraged quickly. Conversely, market dysfunction may cause issues with Bitcoin fungibility and where divergences among various markets are slow to "heal". Thus, our rolling-correlation analyses allow us to spot relatively high (low) periods of cross-market functioning when said rolling-correlations are relatively high (low).

Table 1. Summary Statistics for Price Data

\begin{tabular}{|c|c|c|c|c|}
\hline Price & Mt.Gox & Bitstamp & BTC-E & Bitfinex \\
\hline Average & 105.07 & 289.43 & 120.08 & 231.99 \\
\hline Std. Deviation & 234.07 & 267.04 & 231.51 & 260.01 \\
\hline Obsv. period & $7 / 17 / 10-2 / 25 / 14$ & $9 / 13 / 11-9 / 30 / 14$ & $8 / 14 / 11-9 / 30 / 14$ & $3 / 31 / 13-9 / 30 / 14$ \\
\hline
\end{tabular}

Table 2. Summary Statistics for Volume Data

\begin{tabular}{|c|c|c|c|c|}
\hline Price & Mt.Gox & Bitstamp & BTC-E & Bitfinex \\
\hline Average & 42295 & 8484 & 6885 & 10518 \\
\hline Std. Deviation & 47402 & 12256 & 11201 & 10654 \\
\hline Obsv. period & $7 / 17 / 10-2 / 25 / 14$ & $9 / 13 / 11-9 / 30 / 14$ & $8 / 14 / 11-9 / 30 / 14$ & $3 / 31 / 13-9 / 30 / 14$ \\
\hline
\end{tabular}

Further, to fully understand the extent of cross-market informational efficiency, we employ Generalized Impulse Response Functions (Pesaran and Shin, 1998) derived from the following Vector Autoregression (VAR) model

$$
r_{i, t}=\alpha_{0, i}+\beta_{1, i} r_{1, t-1}+\beta_{2, i} r_{2, t-1}+\beta_{3, i} r_{3, t-1}+\beta_{4, i} r_{4, t-1}+\varepsilon_{i, t}
$$

where $r_{i, t}$ represents the log-returns in market $\mathrm{i}$ at time $\mathrm{t}$ and $\beta_{i}$ represents the elasticity between the returns. This equation is estimated separately for each sub-sample based on each market's log-returns (i.e. $r_{i, t}$ ). Note that, unlike traditional Impulse Response Functions, GIRF's are unaffected by variable ordering in the VAR specification and estimation process. As such, our results are free from potentially-biasing variable ordering decisions or explicitlydefined structure in each VAR's variance-covariance matrix.

During periods of relatively-high cross-market functioning, we should generally expect to see a high level of crossmarket informational efficiency where shocks from one (related) market are quickly incorporated into the price of another (related) market. As our GIRF analyses elicit the time-path of a unit-standard deviation shock from Bitcoin market $i$ to Bitcoin market $j$, we can determine periods of relatively high (low) levels of market functioning when the time-to-cross market efficiency is low (high). Consequently, by comparing the GIRF time-paths among the three, previously defined sub-samples, we'll be able to elicit the relative level of cross-market efficiency and functioning.

\section{RESULTS}

In this section, we describe our results from the graphical analysis and the Impulse Response function analysis we 
conduct. Being a commodity with a relatively short history of being traded, we hope that with the following, we will be able to understand how robust and resilient Bitcoin exchanges are to exogenous shocks.

\section{Price, Market Share, \& Distortions}

In order to understand the results of our graphical and Impulse Response Function analyses, it is instructive to first describe price dynamics of Bitcoin and the market positions of the different exchanges over the time period that we consider.

Figure 1 plots the weighted price for the Bitcoin/US Dollar exchange rate for the entire sample length of 7/17/2010 to 9/30/2014. It is clearly visible that two periods describe our sample: the pre-2013 period with lower prices and the post-2013 period with higher prices.

But despite the fact that the price can be categorized into two periods, volatility has been persistently high in Bitcoin markets. Whereas the price fluctuated around numbers below $\$ 1$ before 2011, it saw a hundred-fold increase during that year before returning to its previous value. The price peaked to its highest level around 2013 when it reached $\$ 1242$ on November 2013. Since then, the price has experienced sharp fluctuations and decreased to levels around \$230 as of September 2015.

The Weighted Price Index is constructed as a weighted average of all available markets' (e.g. Mt. Gox, Bitfinex, Bitstamp, and BTC-e; where available) closing Bitcoin/USD prices where dollar volume is used as the weighting mechanism.

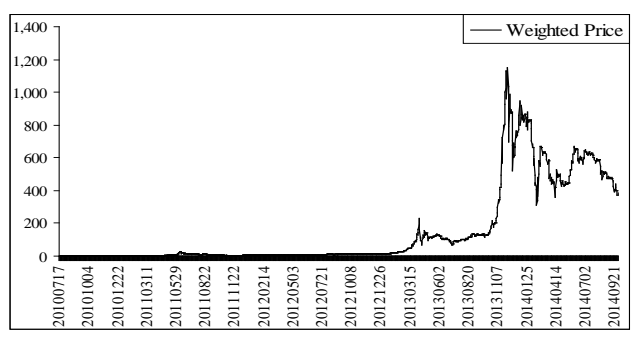

Figure 1. Weighted Bitcoin Price

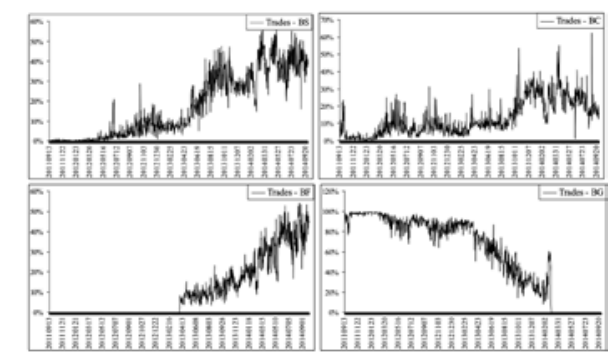

Figure 2. Trade Shares by Market

As mentioned in the introduction and as can be seen in Figure 2, since the inception of Bitcoin as a virtual currency, the most dominant exchange on which Bitcoin were traded was Mt. Gox. Until the first half of 2013, the vast majority of trades occurred on Mt.Gox and other exchanges that existed during this period, only played a negligible role. This dominant position for Mt. Gox lasted until the first half of 2013, when Bitfinex was introduced and Mt. Gox started facing difficulties related to theft and competition. During the next year, Mt.Gox's market share declined precipitously. In addition, one can see that after Mt.Gox's suspension of trading activities, Bitfinex became the most dominant exchange on which Bitcoin were traded. In the next paragraphs, we will report our findings that in addition to absorbing the market share that Mt.Gox once claimed, the Bitcoin market exhibited resilience and recovered from the volatility created by Mt.Gox’s decline.

Figure 2 sub-graphs plot the Trade Share for each market, where data exists, and for the entire sample length of 7/17/2010 to 9/30/2014. Trade Share for a given market is defined as total daily trading in Bitcoins on that market divided by the total amount of Bitcoin trading across all markets. BS, BC, BF, and BG stand for Bitstamp, BTC-e, Bitfinex, and Mt. Gox, respectively.

Figure 3 plots the total number of Bitcoin trades across all exchanges (e.g. Mt. Gox, Bitstamp, BTC-e and Bitfinex; where available) for the entire sample length of 7/17/2010 to 9/30/2014. 


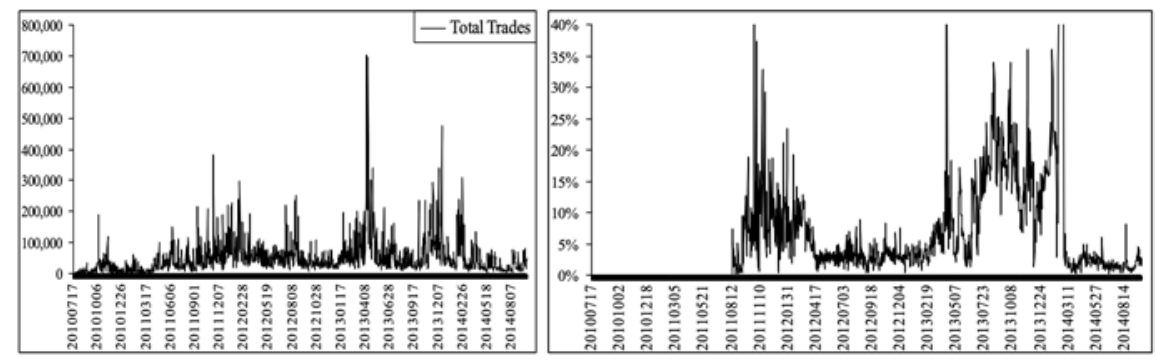

Figure 3. Total Bitcoin Trades Figure 4. Total Bitcoin Pricing Error

In this figure, we can see that since the inception of Bitcoin exchanges, trading volume in Bitcoin markets was generally characterized by high volatility. Despite this continuous volatility, substantial increases in trading volume occurred around August 2011, September 2011 and March 2013. The increase in trading volume during these periods also coincides with the introduction of the rival exchanges BTC-e, Bitstamp, and Bitfinex, which deteriorated Mt. Gox's dominant position, and also with a substantial increase in the price of Bitcoin's, where within a period of only two months, the price increased tenfold to around \$150. During the period of our analysis the market experienced other spikes in volatility, likely as a result of uncertainties surrounding the Bitcoin market, Mt.Gox's deterioration and the introduction of other rival exchanges.

While trading volume by itself does not necessarily have relevant implications that allow us to understand market dynamics, it allows us to infer that exogenous shocks, such as the introduction of rival exchanges or legal issues, result in high volatility in the Bitcoin market.

To understand the effects of these factors, i.e. increasing competition for Mt. Gox and its subsequent decline, on the volatility in the exchanges that we consider, we can look at figures 4 and 5, which describe the Bitcoin Pricing Error for Bitcoin/US Dollar exchange and the Bitcoin Price divergence, respectively.

Total Bitcoin Pricing Error, which is depicted in Figure 4, is the sum of all individual markets' Bitcoin Pricing Error. Individual pricing error for a given market is equal to the absolute divergence between that market's closing price and the average closing price of all available markets (e.g. Mt. Gox, Bitfinex, Bitstamp, and BTC-e), divided by the average closing price of all available markets. The sample period we consider appears to be characterized by two periods of high pricing error and volatility, and by two periods of low pricing error and volatility. Confirming our previous findings, the first period of high error and volatility coincides with the introduction of Bitstamp and BTC-e as two rival exchanges to Mt. Gox. Unsurprisingly, this created a period of high volatility which lasted for several months. After this tumultuous period, a period of calm characterized the Bitcoin market, where the divergence of prices and volatility were relatively low. The second period with high pricing error and high volatility coincides with the period, in which Bitfinex was introduced as the third rival exchange and in which Mt. Gox started experiencing a decline in its dominant market position. This prolonged episode ended after the first part of 2014 when Mt. Gox suspended operations and Bitfinex, Bitstamp and BTC-e remained as the three dominant exchanges. As a result, pricing error and volatility experienced a substantial decline.

In summary, it is clearly visible that the introduction of rival exchanges and the decline of Mt. Gox resulted in severe fluctuations and differences in Bitcoin price across different exchanges. At the same time, pricing error and volatility seem to decrease once the initial shock of the introduction of a new exchange is absorbed by the market. This sequence of events allows us to draw the preliminary inference that Bitcoin exchanges are susceptible to volatility shocks and price distortions, but that these are temporary, such that the market recovers quickly.

This result is confirmed by Figure 5, which again depicts individual pricing error for a given market. Here, individual pricing error increases with the introduction of the non-Mt. Gox exchanges and with Mt. Gox's decline and as such, volatility in all Bitcoin exchanges increased. In contrast to these two periods described above, one can also see that there is a third period after the decline of Mt. Gox, in which there is very low pricing error and divergence exhibited across exchanges that we consider. This increase and subsequent dramatic decline of market volatility confirms our inference that Bitcoin markets are susceptible to shocks, but also exhibit resilience. In the 
following, we perform cross-correlation and Generalized Impulse Response analysis to support this finding.

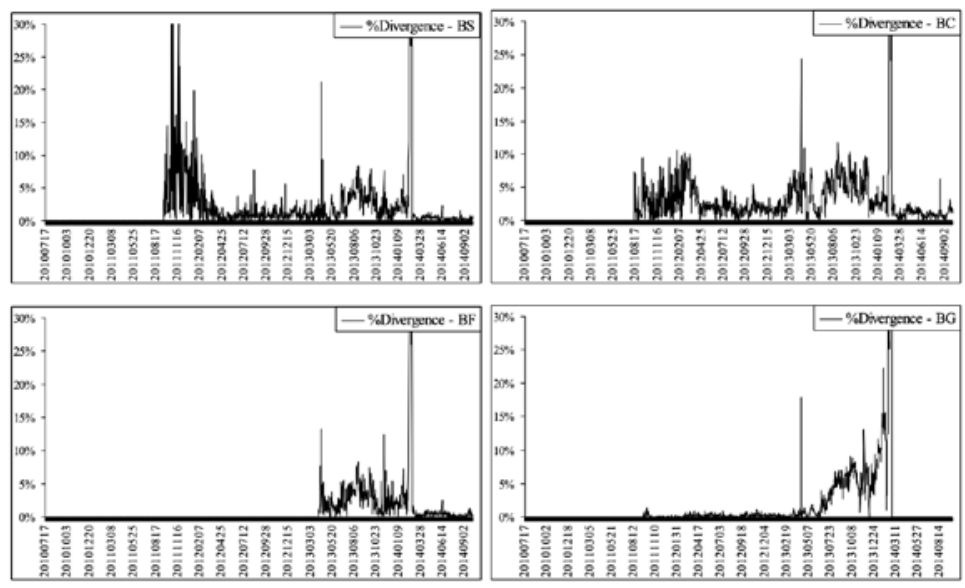

Figure 5. Price Divergence

\section{Cross-Correlation Analysis}

In Figure 6, we graph pair-wise cross-market contemporaneous (close-to-close) returns correlations. Here, we analyze correlations of returns across the four exchanges that we consider. Of the Bitcoin exchanges that we consider, only Mt. Gox, Bitstamp and BTC-e existed prior to 2013, whereas Bitfinex was introduced in March 2013. In order to understand the effect of Mt. Gox's decline as the dominant exchange and the introduction of Bitfinex as a new exchange, on the other markets, we analyze the correlation of returns between the respective exchanges. First, we consider the correlations between Mt. Gox and the remaining exchanges, and then the correlations between the non-Mt. Gox exchanges.

We can see that across all exchanges, correlations of returns with Mt. Gox were volatile until the end of 2012. Following a brief period of relative calm, correlations again declined significantly before Mt. Gox's collapse. In line with this result, one can also see that correlations of returns among non-Mt.Gox exchanges during this period experienced significant volatility.

The effects of the introduction of Bitfinex after March 2013 impacted markets in two ways: there is a brief increase in volatility of correlations across exchanges. While the introduction of Bitfinex did not materially impact correlations among non-Mt.Gox exchanges, it materially impacted correlations to Mt. Gox. However, volatility decreases significantly once Mt. Gox ceases operations and the correlation between returns in the respective exchanges becomes very high. This result reconfirms our initial inference that the Bitcoin market is subject to volatility shocks but eventually gains robustness.

\section{Cross-Market Transmission}

Finally, to analyze cross-market dynamics, we utilize Generalized Impulse Response Function's (GIRF) (reported in return standard deviation responses in market $i$ due to a unit standard deviation shock in the returns of market $j$.). To understand whether there have been different dynamics before Bitfinex was introduced and after Mt. Gox ceased to exist, we conduct our GIRF analysis by dividing our time frame into several intervals: pre- Bitfinex, interval when all markets operated and post-Mt.Gox. 


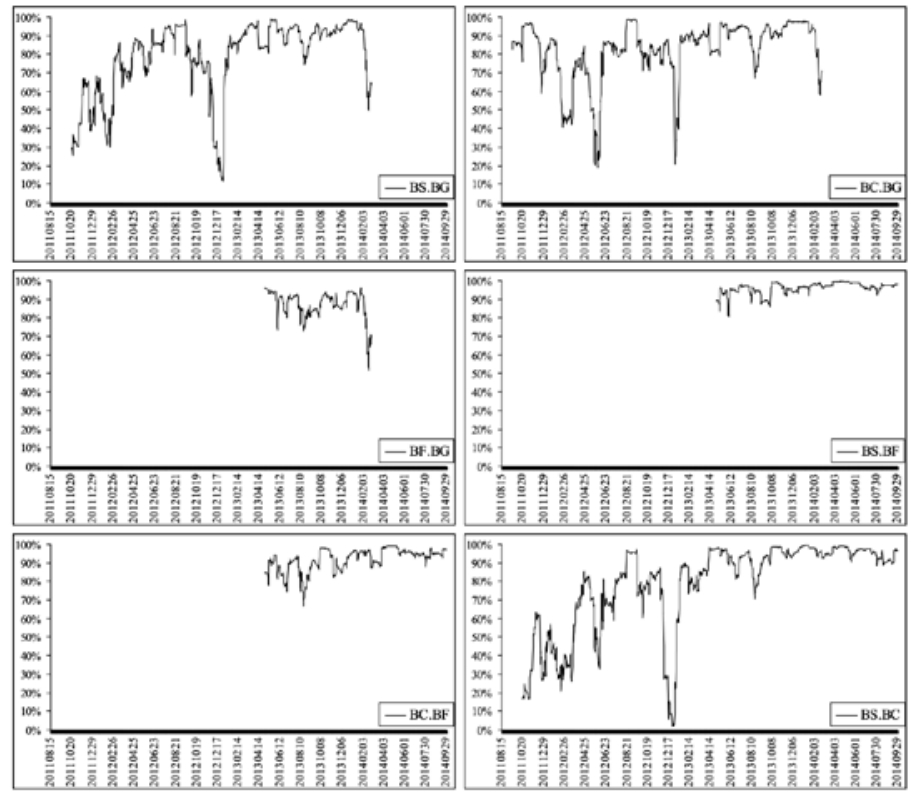

Figure 6. Cross-Market Contemporaneous Returns Correlations

There are several important results that confirm the results from the previous sub-sections. As demonstrated in Figures 7, 8 and 9, the pre-Bitfinex period is characterized by modest responses to returns upon experiencing a shock to the returns in other exchanges. Correspondingly, it is a period of low volatility and relatively high market integration. In contrast to this period, the period in which all exchanges operated, depicted in Figure 8, is characterized by prolonged high volatility and sensitivity to changes in the returns in other exchanges. Finally, GIRF's for the post-Mt.Gox show that the Bitcoin market consolidated quickly after the decline of Mt. Gox, such that shocks to returns in other exchanges did not cause significant volatility in the returns on Bitcoin.

It is important to note that our GIRF analysis corroborates the results discussed in the previous sections. We have used as relevant measures the price of Bitcoins, trade shares and volumes of the exchanges we have considered, and pricing error, price divergence and cross correlations between the returns in the respective exchanges. All of these measures, and our GIRF analysis show that the introduction of rival exchanges resulted in significant spikes in volatility, but that this volatility decreased within a short period of time.

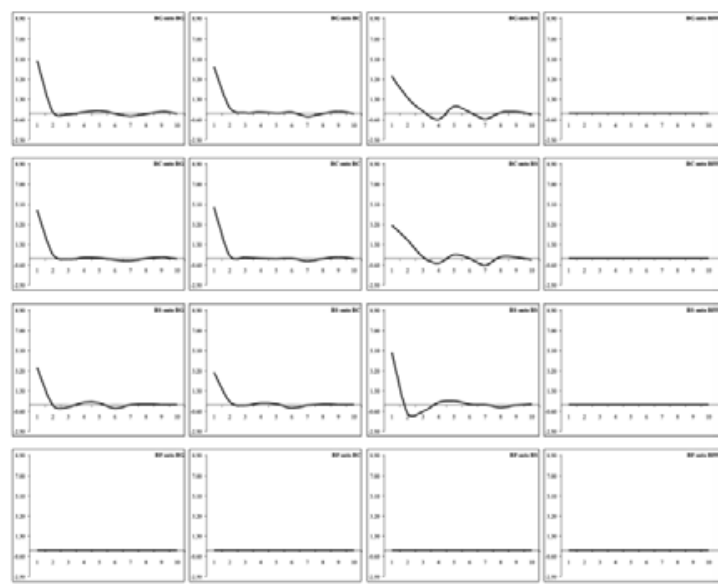

Figure 7. Generalized Impulse Response Functions (Pre-Bitfinex)

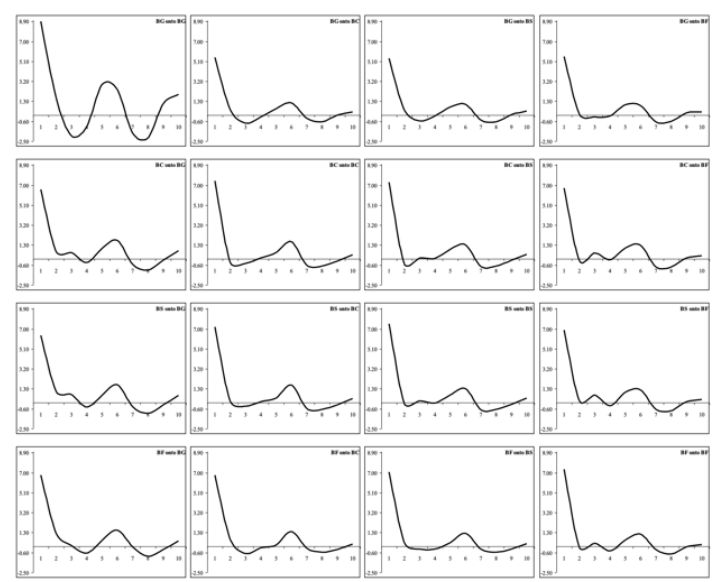

Figure 8. Generalized Impulse Response Functions (All Markets) 


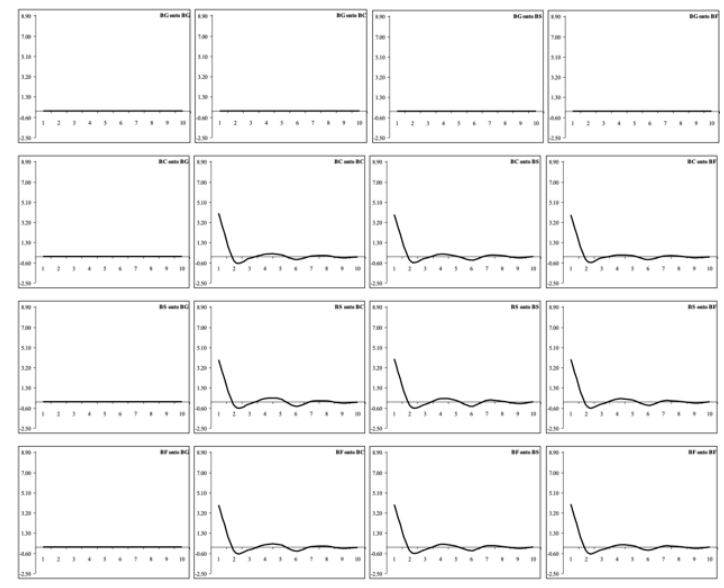

Figure 9. Generalized Impulse Response Functions (Post-Mt. Gox)

\section{SUMMARY}

Our findings show that Bitfinex's introduction led to a shifting of trading activity from the once popular Mt. Gox exchange to other exchanges, in particular, Bitfinex. Mt. Gox's loss of trading activity caused price distortions in all Bitcoin markets under study. In addition, cross-market relationships, especially those between Mt. Gox and other markets, became less efficient while Mt. Gox as in its death throes. These inefficiencies, however, disappeared once Mt. Gox ceased to exist. Relevant implications of our results include that Bitcoin markets are susceptible to volume losses to rival exchanges, that shifting trading activity is also associated with price distortions mainly originating from the suffering exchange, but that Bitcoin cross-market dynamics are resilient and, ultimately, self-healing from shocks. Evidence of the resiliency of the Bitcoin market is good news for the future of cryptocurrencies, including its organizational and individual users and future adopters.

\section{REFERENCES}

Abrams, R., \& Popper, N. (2014, Feb 26). Trading Site Failure Stirs Ire and Hope for Bitcoin. The New York Times. http://nyti.ms/1euGCim

Böhme, R., Christin, N., Edelman, B., \& Moore, T. (2015). Bitcoin: Economics, technology, and governance. Journal of Economic Perspectives, 29(2), 213-38.

Feder, A., Gandal, N., Hamrick, J. T., \& Moore, T. (2017). The impact of DDoS and other security shocks on Bitcoin currency exchanges: Evidence from Mt. Gox. Journal of Cybersecurity, 3(2), 137-144.

Gandal, N., Hamrick, J. T., Moore, T., \& Oberman, T. (2018). Price manipulation in the Bitcoin ecosystem. Journal of Monetary Economics, 95, 86-96.

Hill, K. (2014). The Bitcoin Economy's 'Backbone' Is Bitstamp, An Exchange Run By Two Young Slovenians. Forbes. Retrieved September 12, 2016, from http://www.forbes.com/sites/kashmirhill/2014/06/26/BitcoinBitstamp/

Pesaran, M.H., \& Shin, Y. (1998). Generalized impulse response analysis in linear multivariate models. Economics Letters, 58(1), 17-29. 